\title{
Local Aspects of Superselection Rules
}

\author{
Sergio Doplicher \\ Istituto Matematico G. Castelnuovo, Università di Roma, I-00185 Roma, Italy
}

\begin{abstract}
We study a theory of short range forces in terms of local observable quantities; among the superselection structure determined by the algebra of all local observables, to each additive independent charge we associate local observables having a meaning analogous to the regularized integrals of charge density fields over a finite volume. Among other assumptions, we require that parastatistics are absent from the theories considered.
\end{abstract}

\section{Introduction}

The central role of the principle of locality in quantum field theory has long been stressed by Haag [1]. As formulated in [2] by this principle it is meaningful to consider (the algebra generated by) all the observables in a given theory that can be measured within a fixed bounded space-time region; moreover the correspondence so obtained

$$
\mathcal{O} \rightarrow \mathfrak{U}(\mathcal{O})
$$

between regions and algebras of observables should contain all the physical information about the theory. In other words, the relevant specification on local observables is their spacetime localization and not their particular interpretation $[1,2]$.

This point of view proved to be extremely fruitful over the years, in analyzing matters of principles and the general structure of quantum field theory. In particular it has been possible to see how important features of a theory, that are usually described in terms of non-observable quantities like charged fields, are actually determined by the correspondence (1.1) alone.

We refer e.g. to the features of particle statistics and superselection structure. The superselection quantum numbers appear as labels of the equivalence classes of special irreducible representations of the algebra of all local observables, and are global data; they can be viewed as eigenvalues of "charge" operators $Q, a$ posteriori determined by the algebra of quasilocal observables but not belonging 
to it. These charge operators are the generators of gauge transformations of the first kind, which leave pointwise invariant the algebra of observables but act on the field algebra determined by it [3-5].

In Lagrangian field theory, an internal symmetry leaving the Lagrangian invariant determines a conserved current $j_{\mu}$ by Noether's theorem and considering the regularized integral of $j_{0}$ over a finite volume

$$
\dot{j}_{0}\left(f_{R} g_{\delta}\right)=\int \dot{j}_{0}(\mathbf{x}, t) f_{R}(\mathbf{x}) g_{\delta}(t) d^{3} x d t
$$

[where $f_{R}, g_{\delta}$ are nonnegative smooth functions with compact support, $f_{R}(\mathbf{x})=1$ for $|\mathbf{x}| \leqq R$ and $g_{\delta}(t)=0$ for $\left.|t| \geqq \delta, \int g_{\delta}(t) d t=1\right]$, we obtain local charge operators which as $R \rightarrow \infty$, approach the global charge $Q$ in the sense that

$$
Q F \Omega=\lim _{R \rightarrow \infty}\left[j_{0}\left(f_{R} g_{\delta}\right), F\right] \Omega,
$$

where $\Omega$ denotes the vacuum state vector and $F$ is any quasilocal field operator. Equation (1.3) is a simple consequence of the basic property of the operators (1.2): if $\psi$ is a field operator localized near the origin and destroying $n$ units of the charge considered, then $j_{0}\left(f_{R} g_{\delta}\right) \psi-\psi_{j_{0}}\left(f_{R} g_{\delta}\right)=-n \psi$, or equivalently

$$
\exp \left(i \theta_{j_{0}}\left(f_{R} g_{\delta}\right)\right) \psi \exp \left(-i \theta_{j_{0}}\left(f_{R} g_{\delta}\right)\right)=e^{-i n \theta} \psi \text {. }
$$

By Eq. (1.4) the operator (1.2) is a generator of a unitary group inducing gauge transformations of the first kind on a specific local field algebra.

In this paper we propose to associate to the global superselection quantum numbers $\xi$ determined by a local quantum theory $\mathcal{O} \rightarrow \mathfrak{U}(\mathcal{O})$ local observables $J_{\xi}$ having the same properties (1.3) and (1.4) of the operators (1.2). By this procedure one proves the existence of local measurements with specific physical interpretation $^{1}$. This is a step on the way to proving the existence of local Wightman fields $j_{0}^{\xi}(x)$ having the meaning of charge density for the superselection quantum number $\xi$.

In a Lagrangian theory the operator (1.2) is an observable only if the gauge group is abelian; accordingly we will assume that no superselection sector obeys a parastatistics $[4,6]$. Then to each additive independent charge there will be operators $J_{\xi}$ and we show that they can be chosen to commute with one another; a simultaneous measurement of all of them is possible, determining the charge contained in a region $\mathcal{O}_{1}$ by a measurement in a larger region $\mathcal{O}_{2}$.

In a theory of strictly localizable charges with parastatistics a similar discussion should be possible; since the gauge group would not be abelian, the operators $J_{k}$ would not be observables, but should generate a local current algebra.

We will need several assumptions described in detail below. The most severe restriction from the physical point of view is the limitation to strictly localizable charges, described in $[3,4]$. The present scheme is not applicable to abelian gauge theories or theories with massless particles. In a massive nonabelian gauge theory it is possible that charges enjoy a poorer localizability property [5]. An example of

1 There is an obvious duality between these measurements and the operations defined by charge transfer observables [4, I, Sect. 3] 
our charges would be the baryon number in the old meson theory (and presumably also in QCD). However we feel that the restricted framework of this paper is the natural case to study first.

The local charge operators $J_{\xi}$ are located in the relative commutant $\mathfrak{A}(\mathcal{O})^{c}$ of $\mathfrak{U}(\mathcal{O})$ in the $C^{*}$-algebra $\mathfrak{A}$ generated by all local observables. The subalgebra $\mathfrak{A}\left(\mathcal{O}^{\prime}\right)$ generated by the observables spacelike separated from $\mathcal{O}$ is dense in $\mathfrak{A}(\mathcal{O})^{c}$ [assumption (1) below] but $J_{\xi}$ is not in $\mathfrak{A}\left(\mathcal{O}^{\prime}\right)$. Actually under a natural conjecture (Sect. 5) it follows that $\mathfrak{U}(\mathcal{O})^{c}$ is generated by $\mathfrak{U}\left(\mathcal{O}^{\prime}\right)$ and the $J_{\xi}$. This is in agreement with a proposal made long ago by Haag [7] that gauge theories (of the second kind) should have as a characteristic feature $\mathfrak{U}(\mathcal{O})^{c}=\mathfrak{U}\left(\mathcal{O}^{\prime}\right)$. By [3, I, Sect. 5] this condition amounts to absence of superselection rules associated to localizable charges.

As mentioned at the beginning our main postulate is locality: there is an inclusion preserving correspondence

$$
\mathcal{O} \rightarrow \mathfrak{U}(\mathcal{O})
$$

between $\mathcal{O} \in \mathscr{K}$ (the set of all double cones in Minkowski space; $\mathcal{O} \in \mathscr{K}$ if $\mathcal{O}=x$ $\left.-\bar{V}^{+} \cap y+\bar{V}^{+}, x-y \in V^{+}\right)$and von Neumann algebras acting on a separable Hilbert space $\mathscr{H}_{0}$ (the vacuum Hilbert space). The algebra $\mathfrak{A}(\mathcal{O})$ is generated by local observables in $\mathcal{O}$ and the quasilocal algebra $\mathfrak{A}$ is the $C^{*}$-algebra generated by the union of $\mathfrak{A}(\mathcal{O}), \mathcal{O} \in \mathscr{K}$.

For the sake of extending the correspondence (1.5) to arbitrary regions we introduce on $\mathfrak{A}$ a topology $\mathscr{T}$ which we regard as the natural one for dealing with local normality. $\mathscr{T}$ is the strongest among the locally convex topologies on $\mathfrak{U}$ which are weaker than the norm topology and weaker than the inductive limit of the ultraweak topologies of $\mathfrak{U}(\mathcal{O}), \mathcal{O} \in \mathscr{K}^{2}$. For any $S \subset \mathbb{R}^{4}$ define

$$
\mathfrak{U}(S)=V^{\mathscr{T}}\{\mathfrak{U}(\mathcal{O}) / \mathcal{O} \in \mathscr{K}, \mathcal{O} \subset S\}
$$

where $\bigvee^{\mathscr{T}} M_{\alpha}$ means the $\mathscr{T}$-closed subalgebra of $\mathfrak{U}$ generated by $M_{\alpha} \subset \mathfrak{U}$.

Our assumptions on the correspondence (1.5) are the following

(i) Duality: $\mathfrak{U}(\mathcal{O})=\mathfrak{U}\left(\mathcal{O}^{\prime}\right)^{\prime}, \mathcal{O} \in \mathscr{K}$, which embodies Einstein causality and maximality of the net $\mathfrak{A}(\mathcal{O})\left(\mathcal{O}^{\prime}\right.$ is the open set of all points spacelike to $\left.\mathcal{O}\right)$.

(ii) Additivity: if $\mathcal{O}, \quad \mathcal{O}_{1}, \ldots, \mathcal{O}_{n} \in \mathscr{K}$ and $\mathcal{O} \subset \bigcup_{i=1, \ldots, n} \mathcal{O}_{i}$, then $\mathfrak{U}(\mathcal{O}) \subset \bigvee_{i=1, \ldots, n}^{w^{*}} \mathfrak{U}\left(\mathcal{O}_{i}\right)$

(iii) Time Slice Axiom $[1,9]$ : if $S=\left\{x \in \mathbb{R}^{4} / x_{\mu} \eta^{\mu} \in(a, b)\right\}$ with $\eta \in \mathbb{R}^{4}$ timelike and $a<b$, then

$$
\mathfrak{U}(S)=\mathfrak{U} \text {. }
$$

(iv) Translation Covariance and Spectrum Condition: there is a continuous unitary representation $\mathscr{U}_{0}$ of $\mathbb{R}^{4}$ on $\mathscr{H}_{0}$, a unit vector $\Omega \in \mathscr{H}_{0}$, such that $\sigma\left(\mathscr{U}_{0}\right) \subset \bar{V}^{+}$, $\mathscr{U}_{0}(a) \Omega=\Omega, a \in \mathbb{R}^{4}$ and for each $\mathcal{O} \in \mathscr{K}, a \in \mathbb{R}^{4}$

$$
\mathscr{U}_{0}(a) \mathfrak{U}(\mathcal{O}) \mathscr{U}_{0}(a)^{-1}=\mathfrak{U}(\mathcal{O}+a)
$$

2 Cf. $[8,3.14$ and 3.15]; properties of the topology $\mathscr{T}$ will be discussed elsewhere 
i.e. $\mathscr{U}_{0}(a)$ induces the translation automorphisms $\alpha_{a}$ on $\mathfrak{A}$. The vector state $\omega_{0}$ induced by $\Omega$ on $\mathfrak{A}$ (vacuum state) is the only $\alpha$-invariant normal state, i.e. $\mathfrak{A}$ is irreducible.

As described by $[3,4]$ in our case the superselection structure is described by covariant localized morphisms of $\mathfrak{A}$. A localized morphism $\varrho$ leads to a sector $\xi$ obeying the ordinary Bose or Fermi statistics iff $\varrho$ is an automorphism.

We restrict to theories for which:

a) All localized morphisms are covariant and can be localized in any double cone by a unitary equivalence.

b) There is no sector obeying a parastatistics.

c) There are countably many superselection sectors.

By a) our charges are transportable and carry no minimal length preventing their localization in small regions. Point b) says that the set of superselection sectors is the dual $\hat{\mathscr{G}}$ of a compact abelian gauge group $\mathscr{G}$; by c) $\mathscr{G}$ is separable.

To $\mathfrak{A}$ we can associate a normal field group $\mathscr{F}$, unique up to equivalence, which determines a net $\mathcal{O} \rightarrow \mathfrak{F}(\mathcal{O})$, of field algebras fulfilling normal commutation relations at spacelike distances $[3, \mathrm{II}]$. By assumption a) we have for each $\mathcal{O} \in \mathscr{K}$ the twisted duality property [3, II, Proposition 6.2]

$$
\mathfrak{F}(\mathcal{O})^{\prime}=\mathfrak{F}^{t}\left(\mathcal{O}^{\prime}\right)^{-}
$$

Our last assumption is more technical, and is related to a conjecture of Borchers :

(v) If $\mathcal{O}_{1}, \mathcal{O}_{2} \in \mathscr{K}, \mathcal{O}_{1} \subset$ Interior $\left(\mathcal{O}_{2}\right)$, there is a type I factor $\mathfrak{N}$ such that

$$
\mathfrak{F}\left(\mathcal{O}_{1}\right) \subset \mathfrak{N} \subset \mathfrak{F}\left(\mathcal{O}_{2}\right) \text {. }
$$

This property is known to hold for free fields [10-12]. It is elementary (cf. Sect. 5) that it implies the analogous property for the net of observables:

$\left(\mathrm{v}^{\prime}\right)$ If $\mathcal{O}_{1}, \mathcal{O}_{2} \in \mathscr{K}, \mathcal{O}_{1} \subset$ Interior $\left(\mathcal{O}_{2}\right)$, there is a type I factor $\mathfrak{M}$ such that

$$
\mathfrak{U}\left(\mathcal{O}_{1}\right) \subset \mathfrak{M} \subset \mathfrak{U}\left(\mathcal{O}_{2}\right) .
$$

Unlike our other assumptions we will specify when we use $(v)$ or $\left(v^{\prime}\right)$; we feel that our results should be freed from these technical assumptions.

Assumption (v) is tightly connected with local implementation of symmetries. It is implied by the local implementability of the flip $\psi_{1} \otimes \psi_{2} \rightarrow \psi_{2} \otimes \psi_{1}$ in the theory $\mathfrak{F} \otimes \mathfrak{F}[12]$ and implies that any isomorphism $\sigma$ of $\mathfrak{F}\left(\mathcal{O}_{1}\right)$ onto $\mathfrak{F}\left(\mathcal{O}_{2}\right), \mathcal{O}_{1}$, $\mathcal{O}_{2} \in \mathscr{K}$, is implemented by a unitary in some $\mathfrak{F}\left(\mathcal{O}_{0}\right)$, by the implementation theorem [15, Chap. III, Sect. 8, Corollary 8] (see e.g. [10]). Specifically if $\mathcal{O}_{1}$ CInterior $\left(\mathcal{O}_{2}\right)$ to each gauge transformation $g \in \mathscr{G}$, there is a unitary $V_{g} \in \mathfrak{F}\left(\mathcal{O}_{2}\right)$ such that

$$
\text { Ad } V_{g} \mid \mathfrak{F}\left(\mathcal{O}_{1}\right)=\alpha_{g}, \quad \text { i.e. } \quad V_{g} \psi V_{g}^{-1}=\langle\overline{\xi, g}\rangle \psi
$$

for each field operator $\psi \in \mathfrak{F}\left(\mathcal{O}_{1}\right)$ destroying the charge $\xi(\xi \in \hat{\mathscr{G}}, g \in \mathscr{G} \rightarrow\langle\xi, g<$ is the duality between $\mathscr{G}$ and $\hat{\mathscr{G}}$ ).

Our problem is first to show that we can choose $V_{g}$ gauge invariant, i.e. $V_{g} \in \mathfrak{U}\left(\mathrm{O}_{2}\right)$; furthermore, if we have continuous homomorphisms $\theta \in \mathbb{R} \rightarrow e^{i \theta} \in \mathbb{T} \rightleftarrows g_{\theta} \in \mathscr{G}$, we have to choose $V_{g \theta}$ to be a continuous one parameter 
group. Then by the Stone theorem $V_{g \theta}=e^{i J \theta}$ and $J$ is the desired local charge operator.

By using the powerful results of [16] we can even choose $V_{g}$ to be a strongly continuous representation of $\mathscr{G}$ so that, by such a special choice the operator $J$ has pure point spectrum, the integers; this is different from the operator (1.2).

If the operators $V_{g}$ belong to $\mathfrak{U}\left(\mathcal{O}_{2}\right)$ by (1.9) we have

$$
V_{g} \in \mathfrak{U}\left(\mathcal{O}_{1}\right)^{\prime} \cap \mathfrak{U}\left(\mathcal{O}_{2}\right), \quad \psi V_{g} \psi^{-1}=\langle\xi, g\rangle V_{g}
$$

for each unitary field operator $\psi \in \mathfrak{F}\left(\mathcal{O}_{1}\right)$ destroying the charge $\xi$.

In view of Eq. (1.10) we have to study the action on $\mathfrak{U}\left(\mathcal{O}_{1}\right)^{\prime} \cap \mathfrak{U}\left(\mathcal{O}_{2}\right)$ of the automorphisms of $\mathfrak{U}$ localized in $\mathcal{O}_{1}$. It is easily seen that this action factors through an action $\tau$ of the group $\hat{\mathscr{G}}=\Gamma / \mathscr{I}$ of superselection sectors [3, II]. This fact is discussed in Sect. 2. Existence of a unitary $V_{g}$ fulfilling (1.10) for each $g \in \mathscr{G}$ is a strong property of $\tau$ called dominance by Connes and Takesaki [16]. In Sect. 3 we show that $\tau$ is dominant if $(v)$ holds. In Sect. 4 we discuss the properties of local charge operators when $\tau$ is dominant. In Sect. 5 we comment upon the assumptions $(\mathrm{v}),\left(\mathrm{v}^{\prime}\right)$ and interpret some of our results as Galois relations.

\section{The Action $\tau$ of the Group of Superselection Sectors}

Denote by $\Gamma(\mathcal{O})$ (respectively $\mathscr{I}(\mathcal{O})$ ) the group of automorphisms (respectively inner automorphisms) $\gamma$ of $\mathfrak{A}$ localized in $\mathcal{O}$, i.e. $\gamma \mid \mathfrak{U}\left(\mathcal{O}^{\prime}\right)=$ identity; let $\mathscr{I}=\bigcup_{\mathcal{O} \in \mathscr{K}} \mathscr{I}(\mathcal{O})$, $\Gamma=\bigcup_{\mathcal{O} \in \mathscr{K}} \Gamma(\mathcal{O})$. By assumption a) in the Introduction, for each $\mathcal{O} \in \mathscr{K}$ we have a section

$$
\xi \in \hat{\mathscr{G}}=\Gamma / \mathscr{I} \rightarrow \gamma_{\xi} \in \Gamma(\mathcal{O}),
$$

then for each pair $\xi, \xi^{\prime} \in \Gamma / \mathscr{I}$ there is $\sigma_{\xi, \xi^{\prime}} \in \mathscr{I}(\mathcal{O})$ such that

$$
\gamma_{\xi} \gamma_{\xi^{\prime}}=\sigma_{\xi, \xi^{\prime}} \gamma_{\xi \xi^{\prime}}
$$

and setting

$$
\tau_{\xi}^{\mathcal{O}} \equiv \gamma_{\xi} \mid \mathfrak{U}(\mathcal{O})^{c}
$$

we get a (section independent) homomorphism $\tau^{\mathcal{O}}$ of the commutative discrete group $\hat{\mathscr{G}}$ into $\operatorname{Aut}\left(\mathfrak{H}(\mathcal{O})^{c}\right)$.

2.1. Remark. If $\mathcal{O}_{1}, \mathcal{O}_{2} \in \mathscr{K}$ have nonempty common interiors, $\tau^{\mathcal{O}_{1}}$ and $\tau^{\mathcal{O}_{2}}$ coincide on $\mathfrak{U}\left(\mathcal{O}_{1}\right)^{c} \cap \mathfrak{U}\left(\mathcal{O}_{2}\right)^{c}$.

It suffices to define $\tau^{\mathscr{O}_{1}}$ and $\tau^{\mathcal{O}_{2}}$ using a section (2.1) with $\gamma_{\xi}$ localized in $\mathcal{O}_{0} \in \mathscr{K}$, $\mathrm{O}_{0} \subset \mathrm{O}_{1} \cap \mathrm{O}_{2}$.

2.2. Notation. With $\mathcal{O}_{1}, \mathcal{O}_{2} \in \mathscr{K}$ we write $\mathcal{O}_{1} \subset \subset \mathcal{O}_{2}$ if $\mathcal{O}_{1} \subset$ Interior $\left(\mathcal{O}_{2}\right)$. For each $x \in \mathbb{R}^{4}$ call $I_{x}$ the set of pairs $w=\left(\mathcal{O}_{w}, \tilde{\mathcal{O}}_{w}\right), \mathcal{O}_{w}, \tilde{\mathcal{O}}_{w} \in \mathscr{K}$, such that $\mathcal{O}_{w} \subset \subset \tilde{\mathcal{O}}_{w}$ and $x \in \operatorname{Interior}\left(\mathcal{O}_{w}\right)$. If $u, w \in I_{x}$ we write $u<w$ if $\tilde{O}_{w} \subset \subset \mathcal{O}_{u} \subset \subset \tilde{\mathcal{O}}_{u} \subset \subset \tilde{\mathcal{O}}_{w}$. 
To each point $x \in \mathbb{R}^{4}$ we associate a $C^{*}$-algebra $\mathfrak{U}_{x}^{*}$ generated by a net of von Neumann algebras $\left\{\mathfrak{R}_{w} ; w \in I_{x}\right\}$ setting

$$
\begin{aligned}
& \mathfrak{R}_{w}=\mathfrak{U}\left(\mathcal{O}_{w}\right)^{\prime} \cap \mathfrak{U}\left(\tilde{\mathcal{O}}_{w}\right) ; \\
& \mathfrak{I}_{x}=\left\{\bigcup_{w \in I_{x}} \mathfrak{R}_{w}\right\}^{- \text {norm }} .
\end{aligned}
$$

By the Remark 2.1, the actions (2.3) define an action $\tau^{x}$ of $\hat{\mathscr{G}}$ on $\mathfrak{U}_{x}^{c}$ leaving each $\mathfrak{R}_{w}, w \in I_{x}$, globally stable.

By translation covariance we have for each $a \in \mathbb{R}^{4}$

$$
\alpha_{a}\left(\mathfrak{U}_{x}^{c}\right)=\mathfrak{U}_{x+a}^{c} ; \quad \alpha_{a} \tau^{x} \alpha_{a}^{-1}=\tau^{x+a} .
$$

With $\mathfrak{U}_{x} \subset \mathfrak{U}_{x}^{c}$ the $C^{*}$-algebra generated by $\mathfrak{U}\left(\mathcal{O}^{\prime}\right), \mathcal{O} \in \mathscr{K}, x \in$ Interior $(\mathcal{O})$, we have $\mathfrak{U}_{x} \subset\left(\mathfrak{U}_{x}^{c}\right)^{\tau^{x}}$, the $\tau^{x}$-fixed points. Since $\cap\{\mathfrak{U}(\mathcal{O}) / \mathcal{O} \in \mathscr{K}, x \in \operatorname{Interior}(\mathcal{O})\}=\mathbb{C} \cdot I$ by [17], $\mathfrak{U}_{x}$ and $\mathfrak{U}_{x}^{c}$ are irreducible.

Whenever confusion is not possible we will write $\tau$ for $\tau^{x}$.

2.3. Proposition ${ }^{3}$. Assume $\left(\mathrm{v}^{\prime}\right)$. Then for any $w \in I_{x}$ we have

$$
\operatorname{Kernel} \tau \mid \mathfrak{R}_{w}=\{e\} .
$$

Proof. Let $u \in I_{x}, u<w$ and $\mathfrak{M}_{1}, \mathfrak{M}_{2}$ type $I$ factors such that

$$
\mathfrak{U}\left(\mathcal{O}_{u}\right) \subset \mathfrak{M}_{1} \subset \mathfrak{U}\left(\tilde{\mathcal{O}}_{u}\right) \subset \mathfrak{M}_{2} \subset \mathfrak{U}\left(\tilde{\mathcal{O}}_{w}\right) .
$$

With $\gamma \in \Gamma\left(\mathcal{O}_{w}\right), \gamma \mid \mathfrak{U}\left(\mathcal{O}_{w}\right)^{\prime} \cap \mathfrak{U}\left(\tilde{\mathcal{O}}_{w}\right)=$ identify we have to show $\gamma \in \mathscr{I}$. We first show $\gamma\left(\mathfrak{M}_{1}\right)=\mathfrak{M}_{1}$.

If $A \in \mathfrak{M}_{1}, \gamma(A) \in \mathfrak{U}\left(\tilde{\mathcal{O}}_{u}\right) \subset \mathfrak{M}_{2}$. Since $\mathfrak{U}\left(\mathcal{O}_{w}\right) \subset \mathfrak{U}\left(\mathcal{O}_{u}\right) \subset \mathfrak{M}_{1}, \mathfrak{M}_{1}^{\prime} \cap \mathfrak{U}\left(\tilde{\mathcal{O}}_{w}\right) \subset \mathfrak{R}_{w}$ and $\gamma \mid \mathfrak{M}_{1}^{\prime} \cap \mathfrak{A}\left(\tilde{\mathcal{O}}_{w}\right)=$ identity. Then

$$
\gamma(A) \subset\left(\mathfrak{M}_{1}^{\prime} \cap \mathfrak{U}\left(\tilde{\mathcal{O}}_{w}\right)\right)^{\prime} \cap \mathfrak{M}_{2} \subset\left(\mathfrak{M}_{1}^{\prime} \cap \mathfrak{M}_{2}\right)^{\prime} \cap \mathfrak{M}_{2}=\mathfrak{M}_{1} .
$$

Hence $\gamma\left(\mathfrak{M}_{1}\right) \subset \mathfrak{M}_{1}$ and replacing $\gamma$ by $\gamma^{-1}$ we have $\gamma\left(\mathfrak{M}_{1}\right)=\mathfrak{M}_{1}$. Then there is a unitary $U \in \mathfrak{M}_{1}$ such that, setting $\eta: \eta(A)=U A U^{-1}, A \in \mathfrak{A}$, we have

$$
\gamma(A)=\eta(A), \quad A \in \mathfrak{M}_{1} .
$$

By (2.9), $\eta \mid \mathfrak{U}\left(\mathcal{O}_{w}\right)^{\prime} \cap \mathfrak{M}_{1}=$ identity and $U \in\left(\mathfrak{U}\left(\mathcal{O}_{w}\right)^{\prime} \cap \mathfrak{M}_{1}\right)^{\prime} \cap \mathfrak{M}_{1}=\mathfrak{A}\left(\mathcal{O}_{w}\right)$. Therefore $\gamma=\eta$ on $\mathfrak{U}\left(\mathcal{O}_{u}\right) \subset \mathfrak{M}_{1}$ and $\gamma=\eta=$ identity on $\mathfrak{A}\left(\mathcal{O}_{w}^{\prime}\right)$. By the following remark we have $\gamma=\eta$ on $\mathfrak{U}$ and $\gamma \in \mathscr{I}$.

2.4. Remark. If $\mathscr{O}_{1}, \mathcal{O}_{2} \in \mathscr{K}, \mathcal{O}_{1} \subset \subset \mathcal{O}_{2}$ and $\varrho, \eta$ are endomorphisms of $\mathfrak{U}$ coinciding on $\mathfrak{U}\left(\Theta_{2}\right)$ and $\mathfrak{U}\left(\mathcal{O}_{1}^{\prime}\right)$, then $\varrho=\eta$.

Note that $\varrho, \eta$ are locally normal [20] and isometric, then $\varrho-\eta$ is a linear bounded map from $\mathfrak{A}$ to $\mathfrak{B}\left(\mathscr{H}_{0}\right)$ whose restriction to each $\mathfrak{A}(\mathcal{O})$ is ultraweakly continuous. By $[8,3.14$ and 3.15$] \varrho-\eta$ is continuous from $(\mathfrak{H}, \mathscr{T})$ to $\left\{\mathfrak{B}(\mathscr{H} 0), \sigma w^{*}\right\}$. Since $\varrho, \eta$ are morphisms, $\varrho-\eta$ vanishes on the subalgebra generated by $\mathfrak{A}\left(\mathrm{O}_{2}\right)$ and $\mathfrak{U}\left(\mathcal{O}_{1}^{\prime}\right)$, which is $\mathscr{T}$-dense by (ii) and (iii); hence $\varrho=\eta$.

The following facts will be of frequent use.

3 Compare [3, I, Sect. 5]; see also [18] 
2.5. Remark. The fixed point algebra $\mathfrak{R}_{w}^{\tau}, w \in I_{x}$, is properly infinite.

In fact if $\mathcal{O} \in \mathscr{K}, \mathcal{O} \subset \mathcal{O}_{w}^{\prime} \cap \tilde{\mathcal{O}}_{w}$, then $\mathfrak{A}(\mathcal{O}) \subset \mathfrak{R}_{w}^{\tau}$ and $\mathfrak{A}(\mathcal{O})$ is properly infinite [22].

2.6 Proposition. Let $E \in \mathfrak{R}_{w}^{\tau}$ be a nonzero projection and $w<u \in I_{x}$; then

$$
E \sim I \bmod \mathfrak{R}_{u}^{\tau} .
$$

Proof. This is a special case of a theorem of Borchers [14], which applies here since the relative commutant of $\mathfrak{R}_{w}^{\tau}$ in $\mathfrak{R}_{u}^{\tau}$ includes $\mathfrak{U}(\mathcal{O})$ if $\mathscr{O} \in \mathscr{K}, \mathcal{O} \subset \mathcal{O}_{u}^{\prime} \cap \mathcal{O}_{w}$, and since for some neighbourhood $\mathscr{N}(O)$ of $O$ in $\mathbb{R}^{4}$ we have by $(2.6) \mathscr{U}_{0}(a) \mathfrak{R}_{w}^{\tau} \mathscr{U}_{0}(a)^{-1} \subset \mathfrak{R}_{u}^{\tau}$, $a \subset \mathscr{N}(O)$.

The reader will note that the foregoing discussion would also apply with a trivial change in Proposition 2.3 in the presence of parastatistics.

As discussed in the Introduction, our basic problem is the following: given any $w \in I_{x}$, to each $g \in \mathscr{G}$ does there exist a unitary $V_{g} \in \mathfrak{R}_{w}$ such that

$$
\tau_{\xi}\left(V_{g}\right)=\langle\xi, g\rangle V_{g} ; \quad \xi \in \hat{\mathscr{G}}, \text { and } g \in \mathscr{G} \text {. }
$$

According to Connes and Takesaki [16] a continuous action of a separable locally compact abelian group on a von Neumann algebra is called dominant if it has the above property (2.11) and the fixed point subalgebra is properly infinite (cf. Remark 2.5). Such actions have the following properties.

\subsection{Proposition [16]. Let $\tau$ be dominant; then}

(i) $\mathfrak{R}_{w}$ is generated as a von Neumann algebra by the fixed points $\mathfrak{R}_{w}^{\tau}$ and any collection of unitaries $\left\{V_{g} ; g \in \mathscr{G}\right\}$ fulfilling (2.11).

(ii) There is a choice $\mathscr{V}_{g}, g \in \mathscr{G}$ of solutions of (2.11) such that $g \in \mathscr{G} \rightarrow \mathscr{V}_{g}$ is a strongly continuous unitary representation of $\mathscr{G}$.

[Condition (ii) is equivalent to the assertion that $\left\{\Re_{w}, \tau\right\}$ is isomorphic to $\{\mathfrak{M} \times \mathscr{G}, \hat{\theta}\}$, the $w^{*}$ crossed product of a von Neumann algebra $\mathfrak{M}$ by $\mathscr{G}$ equipped with the dual action; $\mathfrak{M}$ can be identified with $\mathfrak{R}_{w}^{\tau}$ and $\theta_{g}$ with $\operatorname{Ad} \mathscr{V}_{g}$.]

We briefly discuss the freedom in the choice of the representation $\mathscr{V}$ of Proposition 2.7, (ii), when $\tau$ is dominant. We define an action $\theta$ of $\mathscr{G}$ on $\mathfrak{R}_{w}^{\tau}$ by $\theta_{g}(T)$ $=\mathscr{V}_{g} T \mathscr{V}_{g}^{-1}, g \in \mathscr{G}, T \in \mathfrak{R}_{w}^{\tau}$; then (see [16, III.3] and [13]) $\left\{\mathfrak{R}_{w}^{\tau}, \theta\right\} \cong\left\{\mathfrak{R}_{w} \times{ }_{\tau} \hat{\mathscr{G}}, \hat{\tau}\right\}$ and $\theta$ is dominant since $\mathfrak{R}_{w}$ is properly infinite. With $g \in \mathscr{G} \rightarrow \mathscr{V}_{g}^{\prime}$ another continuous unitary representation in $\mathfrak{R}_{w}$ fulfilling (2.11), we have that $\mathscr{U}_{g}=\mathscr{V}_{g}^{\prime} \mathscr{V}_{g}^{-1} \in \mathfrak{R}_{w}^{\tau}$ is a $\theta$-cocycle and $\theta_{g}^{\prime}=\operatorname{Ad} \mathscr{U}_{g} \theta_{g}$. By $\left[16\right.$, Corollary 2.4] there is a unitary $\mathscr{U} \in \mathfrak{R}_{w}^{\tau}$ such that

$$
\theta_{g}^{\prime}=\operatorname{Ad} \mathscr{U} \theta_{g} \operatorname{Ad} \mathscr{U}^{-1} \text {. }
$$

Since $\mathscr{U} \in \mathfrak{R}_{w}^{\tau}, w(g)=\mathscr{V}_{g}^{\prime} \mathscr{U}_{g} \mathscr{V}_{g}^{-1} \mathscr{U}^{-1}$ belongs to $\mathfrak{R}_{w}^{\tau}$ and by (2.12) also to $\left(\mathfrak{R}_{w}^{\tau}\right)^{\prime}$. It follows that all choices $\mathscr{V}_{g}$ induce the same action $\theta_{0}$ on the center $\mathfrak{R}_{w}^{\tau} \cap\left(\mathfrak{R}_{w}^{\tau}\right)^{\prime}$; any two choices $\mathscr{V}, \mathscr{V}^{\prime}$ are related by

$$
\mathscr{V}_{g}^{\prime}=w(g) \mathscr{U}_{\mathscr{V}_{g}} \mathscr{U}^{-1}, \quad g \in \mathscr{G}
$$

with $\mathscr{U}$ a unitary in $\mathfrak{R}_{w}^{\tau}$ and $g \in \mathscr{G} \rightarrow w(g)$ a unitary $\theta_{0}$-cocycle in the center of $\mathfrak{R}_{w}^{\tau}$.

If the $\mathfrak{R}_{w}^{\tau}$ are factors [which would follow from a) $\mathfrak{U}(\mathcal{O})$ is a factor, and b) $\Gamma(\tau)=\mathscr{G}$ or equivalently $\theta_{0}$ is trivial] Eq. (2.13) simplifies to

$$
\mathscr{V}_{g}^{\prime}=\left\langle\xi_{0}, g\right\rangle \mathscr{U}_{g} \mathscr{U}^{-1}
$$


where multiplying by a fixed character amounts to adding a constant to the local charge operators.

\section{When is the Action $\tau$ Dominant?}

In this section we show that $\tau$ is dominant if $\hat{\mathscr{G}}$ is finite under assumption $\left(\mathrm{v}^{\prime}\right)$ and for any countable $\hat{\mathscr{G}}$ under assumption (v).

3.1. Definition. For $w \in I_{x}, g \in \mathscr{G}$, define

$$
\begin{gathered}
\mathscr{M}_{w}(g)=\left\{B \in \mathfrak{R}_{w} / \tau_{\xi}(B)=\langle\xi, g\rangle B, \xi \in \hat{\mathscr{G}}\right\}, \\
H_{w}=\left\{g \in \mathscr{G} / \mathscr{M}_{w}(g) \neq\{0\}\right\} .
\end{gathered}
$$

3.2. Lemma. Let $u, w \in I_{x}, u<w$; with $\mathscr{G}\left(H_{u}\right)$ the subgroup of $\mathscr{G}$ generated by $H_{u}$, for each $g \in \mathscr{G}\left(H_{u}\right)$ there is a unitary element in $\mathscr{M}_{w}(g)$.

Proof. If $B \in \mathscr{M}_{u}(g), \tau_{\xi}\left(B^{*} B\right)=B^{*} B$ and $\tau_{\xi}\left(B B^{*}\right)=B B^{*}, \xi \in \mathscr{G}$; hence if $W|B|$ is the polar decomposition of $B$, we have $|B| \in \mathfrak{R}_{u}^{\tau}$ and $W \in \mathscr{M}_{u}(g)$. Since also $W^{*} W$, $W W^{*} \in \mathfrak{R}_{u}^{\tau}$, by Proposition 2.6 there are isometries $V_{1}, V_{2} \in \mathfrak{R}_{w}^{\tau}$ with final projections equal to $W W^{*}, W^{*} W$ respectively; then the operator $V_{1}^{*} W V_{2}$ is a unitary in $\mathscr{M}_{w}(g)$. Since obviously $\mathscr{M}_{w}(g) \mathscr{M}_{w}\left(g^{\prime}\right) \subset \mathscr{M}_{w}\left(g g^{\prime}\right)$ and $\mathscr{M}_{w}(g)^{*}=\mathscr{M}_{w}\left(g^{-1}\right)$, the assertion follows.

\subsection{Proposition. If $\hat{\mathscr{G}}$ is finite and we assume $\left(\mathrm{v}^{\prime}\right)$ then $\tau$ is dominant.}

Proof. By Fourier analysis $\mathfrak{R}_{w}$ is the linear span of $\mathscr{M}_{w}(g), g \in \mathscr{G}$. Hence $\xi_{0} \in \hat{\mathscr{G}}$, $\left\langle\xi_{0}, g\right\rangle 1=$ for each $g \in H_{w}$ implies $\tau_{\xi_{0}}=$ identity on $\mathfrak{R}_{w}$ and by Proposition 2.3 $\xi_{0}=e$. In other words, $H_{w}^{\perp}=\{e\}$ and $\mathscr{G}\left(H_{w}\right)=\mathscr{G}$. By Lemma 3.2, for any $u>w$, $H_{u}=\mathscr{G}$ and each $\mathscr{M}_{u}(g)$ contains a unitary, i.e. $\tau$ is dominant. Since $w$ was arbitrary $\tau$ is dominant on any $\mathfrak{R}_{u}$.

3.4. Proposition. Assume property (v) and that $\mathscr{G}$ is connected. Then $\tau$ is dominant.

Proof. We first extend the action $\tau$ to the field algebra. With $\mathscr{F}$ the normal field group, recall that we have the exact sequence $1 \rightarrow \mathscr{A} \rightarrow \mathscr{F} \rightarrow \hat{\mathscr{G}} \rightarrow e$; under the assumption a) for each double cone we also have the exact sequence $1 \rightarrow \mathscr{A}(\mathcal{O}) \rightarrow \mathscr{F}(\mathcal{O}) \rightarrow \hat{\mathscr{G}} \rightarrow e$; here $\mathscr{A}(\mathcal{O})$ denotes the unitary group in $\mathfrak{A}(\mathcal{O})$ and $\mathscr{A}=\bigcup_{\mathscr{O} \in \mathscr{K}} \mathscr{A}(\mathcal{O})$. We can choose a section $\xi \in \hat{\mathscr{G}} \rightarrow \psi_{\xi} \in \mathfrak{F}\left(\mathcal{O}_{1}\right), \psi_{\xi}$ unitary; then $\psi_{\xi} \psi_{\xi^{\prime}}$ $=u_{\xi, \xi^{\prime}} \psi_{\xi \xi^{\prime}}, u_{\xi, \xi^{\prime}} \in \pi(\mathfrak{U}(\mathcal{O}))^{4}$. Define the action $\tilde{\tau}$ on $\pi\left(\mathfrak{U}\left(\mathcal{O}_{1}\right)\right)^{\prime} \cap \mathfrak{F}\left(\mathcal{O}_{2}\right)$, for $\mathcal{O}_{1} \subset \subset \mathcal{O}_{2}$, by $\tilde{\tau}_{\xi}=A d \psi_{\xi}$. The action $\tilde{\tau}$ is a (section independent) representation of $\hat{\mathscr{G}}$ by automorphisms of $\pi\left(\mathfrak{A}\left(\mathcal{O}_{1}\right)\right)^{\prime} \cap \mathfrak{F}\left(\mathcal{O}_{2}\right)$ whose restriction to $\pi\left(\mathfrak{U}\left(\mathcal{O}_{1}\right)^{\prime} \cap \mathfrak{U}\left(\mathcal{O}_{2}\right)\right)$ is $\pi \tau \pi^{-1}$.

We will drop the symbol $\pi$ when confusion is not possible.

Note that $\tilde{\tau}$ commutes with the gauge transformations $\alpha_{g}$.

4 With $\xi \in \hat{\mathscr{G}} \rightarrow \gamma_{\xi} \in \Gamma$ a section, $\pi=\bigoplus \pi_{0} \circ \gamma_{\xi}$, where $\pi_{0}$ is the vacuum representation, the field algebra acts on $\mathscr{H}_{0}^{\pi}=\bigoplus_{\xi \in \mathscr{G}} \mathscr{H}_{\xi}, \mathscr{H}_{\xi}=\mathscr{H}_{0}$ 
By assumption (v) to each $g \in \mathscr{G}, w \in I_{x}$, there is a unitary $V_{g} \in \mathfrak{F}\left(\tilde{\mathcal{O}}_{w}\right)$ such that

$$
\text { Ad } V_{g} \mid \mathfrak{F}\left(\mathcal{O}_{w}\right)=\alpha_{g}
$$

hence $V_{g} \in \pi\left(\mathfrak{H}\left(\mathcal{O}_{w}\right)\right)^{\prime} \cap \mathfrak{F}\left(\tilde{\mathcal{O}}_{w}\right)$ and applying (3.4) to the section $\psi_{\xi}$ defining $\tilde{\tau}_{\xi}$ we get

$$
\tilde{\tau}_{\xi}\left(V_{g}\right)=\langle\xi, g\rangle V_{g}, \quad g \in \mathscr{G}, \quad \xi \in \hat{\mathscr{G}} .
$$

Hence $\tilde{\tau}$ is dominant and we have to show that its restriction $\tau$ to $\mathfrak{R}_{w}$ is also dominant.

By Proposition (2.7) (ii) we have a continuous unitary representation $g \in \mathscr{G} \rightarrow \tilde{\mathscr{V}}_{g} \in \pi\left(\mathfrak{H}\left(\mathcal{O}_{w}\right)\right)^{\prime} \cap \mathfrak{F}\left(\mathcal{O}_{w}\right)$ fulfilling (3.4). By continuity there is a neighbourhood $\mathscr{N}(e)$ of the identity is $\mathscr{G}$ such that

$$
\left(\Omega, \tilde{\mathscr{V}}_{g} \Omega\right) \neq 0 \quad \text { for } \quad g \in \mathscr{N}(e) .
$$

With $m$ the average over the gauge group action we have $m: \pi\left(\mathfrak{U}\left(\mathcal{O}_{w}\right)\right)^{\prime} \cap \mathfrak{F}\left(\tilde{\mathcal{O}}_{w}\right) \rightarrow \mathfrak{R}_{w}, m \tilde{\tau}_{\xi}=\tau_{\xi} m$; hence setting $T_{g}=m\left(\tilde{\mathscr{V}}_{g}\right), g \in \mathscr{G}$, we have $T_{g} \in \mathscr{M}_{w}(g)$ and $T_{g} \neq 0$ for $g \in \mathscr{N}(e)$ by Eq. (3.5).

Hence $\mathscr{N}(e) \subset H_{w}$; by Lemma 3.2 if $u>w, u \in I_{x}, \mathscr{M}_{u}(g)$ contains a unitary for each $g \in \mathscr{G}\left(H_{w}\right)$; since $\mathscr{G}$ is connected, $\mathscr{G}\left(H_{w}\right)=\mathscr{G}$ and $\tau$ is dominant on $\mathfrak{R}_{u}$; therefore this holds for all $u \in I_{x}$.

3.5. Theorem. Assume property (v). Then $\tau$ is dominant for any countable $\hat{\mathscr{G}}$.

Proof.Denote by $\mathscr{H}_{F}$ (respectively $\mathscr{H}_{B}$ ) the subspace of $\mathscr{H}_{\pi}$ spanned by all $\mathscr{H}_{\xi}$ with $\xi$ a Fermi (respectively Bose) sector, and define

$$
\tilde{\mathscr{M}}_{w}(g)=\left\{F \in \pi\left(\mathfrak{U}\left(\mathcal{O}_{w}\right)\right)^{\prime} \cap \mathfrak{F}\left(\tilde{\mathcal{O}}_{w}\right) / \tilde{\tau}_{\xi}(F)=\langle\xi, g\rangle F, \xi \in \hat{\mathscr{G}}\right\} .
$$

We divide the proof in three steps.

1. $H_{w}=\left\{g \in \mathscr{G} / \tilde{\mathscr{M}}_{w}(g) \Omega \not \subset \mathscr{H}_{F}\right\}$.

Let $B_{g} \in \tilde{\mathscr{M}}_{w}(g)$ be not a Fermi operator. By the Reeh-Schlieder theorem, if $\mathcal{O} \subset \mathcal{O}_{w}^{\prime} \cap \mathcal{O}_{w}$, there is a Bose operator $\psi \in \mathfrak{F}(\mathcal{O})$ such that $\left(\psi \Omega, B_{g} \Omega\right) \neq 0$. Since $\psi \in \mathfrak{F}\left(\mathcal{O}_{w}\right)^{\prime}$, we have $\psi^{*} B_{g} \in \tilde{\mathscr{M}}_{w}(g)$; then $T_{g} \equiv m\left(\psi^{*} B_{g}\right) \neq 0, T_{g} \in \mathscr{M}_{w}(g)$ and $g \in H_{w}$; we proved one inclusion and the opposite one is obvious, and 1. follows.

Let $h_{0} \in \mathscr{G}$ be defined by $\left\langle\xi, h_{0}\right\rangle=+1$ or -1 if $\xi$ is Bose or Fermi respectively.

2. The subset $H_{w} \subset \mathscr{G}$ is a closed and open subgroup and either $H_{w}=\mathscr{G}$ or $G=H_{w} \cdot\left\{e, h_{0}\right\} \sim H_{w} \times \mathbb{Z}_{2}$.

Note that, with $\mathcal{O} \in \mathscr{K}, \mathcal{O} \subset \mathcal{O}_{w}^{\prime} \cap \tilde{\mathcal{O}}_{w}, \psi$ a unitary Fermi operator in $\mathfrak{F}(\mathcal{O})$, we have

$$
\operatorname{Ad} \psi\left|\mathfrak{F}\left(\mathcal{O}_{w}\right)=\alpha_{h_{0}}\right| \mathfrak{F}\left(\mathcal{O}_{w}\right) \text {. }
$$

If $h \notin H_{w}$ and $\mathscr{V}_{h}$ is a unitary in $\tilde{\mathscr{M}}_{w}(h), \mathscr{V}_{h}$ is Fermi and $\psi_{\mathscr{V}_{h}}$ is a unitary Bose operator in $\mathfrak{F}\left(\tilde{\mathcal{O}}_{w}\right)$ inducing $\alpha_{h h_{0}}$ on $\mathfrak{F}\left(\mathcal{O}_{w}\right)$; then $h h_{0} \in H_{w}$ and $\mathscr{G}=H_{w} \cup H_{w} h_{0}$.

If $H_{w}$ and $H_{w} h_{0}$ are not disjoint, the subgroup generated by $H_{w}$ contains $h_{0}$ and then equals $\mathscr{G}$. By Lemma 3.2 and arbitrariness of $w$ then $\tau$ is dominant.

Let $H_{w} \cap H_{w} h_{0}=\emptyset$. If $h \in H_{w}, h h_{0} \notin H_{w}$ and there is a Fermi unitary in $\tilde{\mathscr{M}}_{w}\left(h h_{0}\right)$; by the argument above there is a Bose unitary in $\tilde{\mathscr{M}}_{w}\left(\left(h h_{0}\right) h_{0}\right)=\tilde{\mathscr{M}}_{w}(h)$.

Bose unitaries form a group and, by the multiplication properties of $\tilde{\mathscr{M}}_{w}(g), H_{w}$ is a group. By Proposition $3.4 H_{w} \supset \mathcal{N}(e)$ and $H_{w}$ is open and closed. Since 
$\mathscr{G}=H_{w} \cup H_{w} h_{0}, H_{w} \cap H_{w} h_{0}=\emptyset$, we have the direct product decomposition $\mathscr{G}=H_{w}$ $\cdot\left\{e, h_{0}\right\}$.

3. If $u>w, \mathscr{M}_{u}\left(h_{0}\right) \neq\{0\}$.

If $H_{w} \neq \mathscr{G}, \mathscr{G}=H_{w} \cdot\left\{e, h_{0}\right\}$, and $\hat{\mathscr{G}}=\left\{e, h_{0}\right\}^{\perp} \cdot H_{w}^{\perp}$, where $\left\{e, h_{0}\right\}^{\perp}=\hat{\mathscr{G}}_{B} \sim \hat{H}_{w}$, denoting by $\hat{\mathscr{G}}_{B}$ the subgroup of Bose sectors. $H_{w}^{\perp} \sim\left\{e, h_{0}\right\}^{\wedge} \sim \mathbb{Z}_{2}$, hence there is a Fermi sector $\xi_{0}, \xi_{0}^{2}=e$, such that

$$
\hat{\mathscr{G}}=\hat{\mathscr{G}}_{B}\left\{e, \xi_{0}\right\} ; \quad\left\{e, \xi_{0}\right\}=H_{w}^{\perp} .
$$

Let $u>w$; if $g \in H_{w}, \mathscr{M}_{u}(g)$ contains a unitary operator $\mathscr{V}_{g}$ by Lemma 3.2. Hence the action $\tau \mid \hat{\mathscr{G}}_{B}$ is dominant on $\mathfrak{R}_{u}$, and $\mathfrak{R}_{u}$ is generated by the subalgebra $\mathfrak{B}$ of $\tau \mid \hat{\mathscr{G}}_{B}$-fixed points and by $\left\{\mathscr{V}_{g}, g \in H_{w}\right\}$. Since $\tau_{\xi_{0}}\left(\mathscr{V}_{g}\right)=\mathscr{V}_{g}$ all $g \in H_{w}=\left\{e, \xi_{0}\right\}^{\perp}$, if $\tau_{\xi_{0}}$ is the identity on $\mathfrak{B}$ it follows that $\tau_{\xi_{0}}$ is the identity on $\mathfrak{R}_{u}$ contrary to Proposition 2.3.

Let $B \in \mathfrak{B}, \tau_{\xi_{0}}(B) \neq B$; setting $T_{h_{0}}=B-\tau_{\xi_{0}}(B)$ we have

$$
\tau_{\xi}\left(T_{h_{0}}\right)=\left\langle\xi, h_{0}\right\rangle T_{h_{0}}, \quad \xi \in \mathscr{G} ;
$$

namely $T_{h_{0}} \in \mathscr{M}_{u}\left(h_{0}\right), T_{h_{0}} \neq 0$ and $h_{0} \in H_{u}$.

Since $H_{u} \supset H_{w}, H_{u}=\mathscr{G}$; by Lemma 3.2, $\tau$ is dominant on $\mathfrak{R}_{v}, v>u$, hence on any $\mathfrak{R}_{v}$.

\section{Local Charges}

By the results of the previous section, under the assumptions made in Sect. 1, for each pair of double cones $\mathcal{O}_{1} \subset \subset \mathrm{CO}_{2}$, we have a continuous unitary representation $g \in \mathscr{G} \rightarrow \mathscr{V}_{g} \in \mathfrak{U}\left(\mathcal{O}_{1}\right)^{\prime} \cap \mathfrak{A}\left(\mathcal{O}_{2}\right)$ inducing the gauge transformations on $\mathfrak{F}\left(\mathcal{O}_{1}\right)$. This representation is unique up to the transformation (2.13).

Since $\mathscr{G}$ is abelian and $\mathscr{V}$ is a representation, $\mathscr{V}$ generates an abelian subalgebra of observables in $\mathfrak{U}\left(\mathrm{O}_{2}\right)$, by a simultaneous measurement of which we can determine the charge localized in $\mathcal{O}_{1}$. In the spectral decomposition

$$
\mathscr{V}_{g}=\sum_{\xi \in \hat{G}}\langle\xi, g\rangle E_{\xi}
$$

$E_{\xi} \in \mathfrak{U}\left(\mathcal{O}_{1}\right)^{\prime} \cap \mathfrak{U}\left(\mathcal{O}_{2}\right)$ is nonzero for all $\xi$ and expresses the local property of a state of having exactly charge $\xi$ in $\mathcal{O}_{1}$ plus some specifications in $\mathcal{O}_{1}^{\prime} \cap \mathcal{O}_{2}$, depending upon the choice (4.1) of the solution to our problem. The vacuum state cannot fulfill any such specification by the Reeh-Schlieder theorem.

The representation $\mathscr{V}_{g}$ can be described in terms of local charges in a more familiar way when $\hat{\mathscr{G}}$ is finitely generated. In this case $\hat{\mathscr{G}}$ has independent generators $\eta_{1}, \ldots, \eta_{q}, \xi_{1}, \ldots, \xi_{p}$ with $\eta_{i}^{v_{i}}=e$ and $\xi_{j}$ aperiodic, i.e. the $\eta_{i}$ are multiplicative charges, $\xi_{j}$ additive charges. The correspondence

$$
\left(m_{1}, \ldots, m_{q}, n_{1}, \ldots, n_{p}\right) \rightarrow \eta_{1}^{m_{1}} \ldots \eta_{q}^{m_{q}} \xi_{1}^{n_{1}} \ldots \xi_{p}^{n_{p}}
$$

is an isomorphism of the Kronecker product

$$
\mathbb{Z}_{v_{1}} \times \ldots \times \mathbb{Z}_{v_{q}} \times \mathbb{Z} \times \ldots \times \mathbb{Z}
$$

onto $\hat{\mathscr{G}}$; accordingly we can identify $\mathscr{G}$ with the topological product of the duals

$$
\mathscr{G} \sim \mathbb{Z}_{v_{1}} \times \ldots \times \mathbb{Z}_{v_{q}} \times \mathbb{T} \times \ldots \times \mathbb{T} .
$$


Let $\varepsilon_{i}$ be the first $v_{i}^{\text {th }}$ root of 1 ; with $\xi \in \hat{\mathscr{G}}, g \in \mathscr{G}, \xi \sim\left(m_{1}, \ldots, n_{p}\right), g \sim\left(m_{1}^{\prime}, \ldots, m_{q}^{\prime}\right.$, $\left.e^{i \theta_{1}}, \ldots, e^{i \theta_{p}}\right)$, the duality between $\mathscr{G}$ and $\hat{\mathscr{G}}$ is given by

$$
\langle\xi, g\rangle=\varepsilon_{1}^{m_{1} m_{1}^{\prime}} \ldots \varepsilon_{q}^{m_{q} m_{q}^{\prime}} e^{i n_{1} \theta_{1}} \ldots e^{i n_{p} \theta_{p}} .
$$

By the identification (4.3) we have

$$
\mathscr{V}_{g}=C_{1}^{m_{1}} \ldots C_{q}^{m_{q}} e^{i \theta_{1} J_{1}} \ldots e^{i \theta_{p} J_{p}}, \quad g \in \mathscr{G},
$$

where $C_{i} \in \mathfrak{U}\left(\mathcal{O}_{1}\right)^{\prime} \cap \mathfrak{U}\left(\mathcal{O}_{2}\right)$ is a unitary with $C_{i}^{v_{i}}=I$ and $J_{k} \eta \mathfrak{U}\left(\mathcal{O}_{1}\right)^{\prime} \cap \mathfrak{U}\left(\mathcal{O}_{2}\right)$ is a selfadjoint operator with spectrum equal to $\mathbb{Z}$.

If $\psi_{\xi} \in \mathfrak{F}\left(\mathcal{O}_{1}\right)$ is a field operator destroying the charge $\xi=\eta_{i}^{m_{1}} \ldots \xi_{p}^{n_{p}}$ then [cf. Eq. (1.9)] $\mathscr{V}_{g} \psi_{\xi} \mathscr{V}_{g}^{-1}=\langle\bar{\xi}, g\rangle \psi_{\xi}$ and

$$
\begin{gathered}
C_{i} \psi_{\xi}=\varepsilon_{i}^{-m_{i}} \psi_{\xi} C_{i}, \quad i=1, \ldots, q, \\
J_{k} \psi_{\xi} \supseteqq \psi_{\xi}\left(J_{k}-n_{k} I\right), \quad k=1, \ldots, p .
\end{gathered}
$$
local $\psi$

By letting $\mathcal{O}_{1}=\mathcal{O}_{1, n} \uparrow \mathbb{R}^{4}$ as $n \rightarrow \infty$ in the above choice we get for each quasi-

$$
\begin{gathered}
D_{i} \psi \Omega=\lim _{n \rightarrow \infty} C_{i}^{(n)} \psi C_{i}^{(n)^{*}} \Omega, \\
Q_{k} \psi \Omega=\lim _{n \rightarrow \infty}\left[J_{k}^{(n)}, \psi\right] \Omega,
\end{gathered}
$$

where $D_{i}, Q_{k}$ are the generators of the global representation $\mathscr{U}(g)$ of $\mathscr{G}$, defined by a formula analogous to (4.4).

Note that by acting on a choice $C_{1}, \ldots, J_{p}$ with a space time translation we get a choice for the translated region.

Any set of $p$ commuting self adjoint operators $\tilde{J}_{1}, \ldots, \tilde{J}_{p}$ affiliated to $\mathfrak{U}\left(\mathcal{O}_{1}\right)^{\prime} \cap \mathfrak{U}\left(\mathcal{O}_{2}\right)$ and fulfilling (4.6) have a similar interpretation. We get all such $p$-tuple of operators as follows. Let $\mathscr{G}_{0}$ be the connected component of $e$ in $\mathscr{G}, \tilde{\mathscr{G}}_{0}$ its simply connected covering $\sim \mathbb{R} \times \ldots \times \mathbb{R}$, and $\sigma$ the canonical homomorphism of $\tilde{\mathscr{G}}_{0}$ into $\mathscr{G}$. Fix a reference continuous unitary representation $g \in \mathscr{G} \rightarrow \mathscr{V}_{g} \in \mathfrak{U}\left(\mathcal{O}_{1}\right)^{\prime} \cap \mathfrak{U}\left(\mathcal{O}_{\tilde{\theta}}\right)=\mathfrak{R}$ fulfilling $\tau_{\xi}\left(\mathscr{U}_{g}\right)=\langle\xi, g\rangle \mathscr{U}_{g}, g \in \mathscr{G}$, and define the action $\tilde{\theta}$ of $\tilde{\mathscr{G}}_{0}$ on $\mathfrak{R}^{\tau}$ by $\tilde{\theta}_{g}=\theta_{\sigma(g)}, \theta_{g}=\operatorname{Ad} \tilde{U}_{g} \mid \mathfrak{R}^{\tau}$. The generic choice $\tilde{J}_{1}, \ldots, \tilde{J}_{p}$ are the generators of the unitary representation

$$
g \in \tilde{\mathscr{G}}_{0} \rightarrow \tilde{\mathscr{V}}_{g}=X_{g} \mathscr{V}_{\sigma(g)} \in \mathfrak{R},
$$

where $X_{q}$ is a continuous unitary $\tilde{\theta}$-cocycle in $\mathfrak{R}^{\tau}$. This wider class of operator systems $\tilde{J}_{1}, \ldots, \tilde{J}_{p}$ should be the natural tool in an approximation procedure to construct $p$ Wightman fields having the meaning of charge densities for the additive independent charges $\xi_{1}, \ldots, \xi_{p}$.

\section{Comments on the General Assumptions}

As mentioned in the introduction, the validity of the present analysis is limited to the case of charges which are strictly localizable in bounded regions. (Assuming duality, we also do not discuss broken gauge symmetries or soliton sectors [19].) By the work of Buchholz and Fredenhagen one is led to consider charges localizable at best in spacelike cones [5]. In this case we cannot expect the 
existence of the analogs to the operator (1.2). Actually by [5] the relevant representations are normal on the relative commutants of subalgebras associated to spacelike cones, unlike our case.

We next discuss assumption $(\mathrm{v})$ and $\left(\mathrm{v}^{\prime}\right)$. By twisted duality (1.8), (v) is equivalent to the following: if $\mathcal{O}_{1}, \mathcal{O}_{2} \in \mathscr{K}, \mathcal{O}_{1} \subset \subset \mathcal{O}_{2}$, the linear map defined by

$$
\psi_{1} \psi_{2} \rightarrow \psi_{1} \otimes \psi_{2}, \quad \psi_{1} \in \mathfrak{F}\left(\mathcal{O}_{1}\right), \quad \psi_{2} \in \mathfrak{F}^{t}\left(\mathcal{O}_{2}^{\prime}\right),
$$

is normal [12]. By restriction to $\pi\left(\mathfrak{U}\left(\mathcal{O}_{1}\right)\right) \subset \mathfrak{F}\left(\mathcal{O}_{1}\right), \pi\left(\mathfrak{U}\left(\mathcal{O}_{2}^{\prime}\right)\right) \subset \mathfrak{F}^{t}\left(\mathcal{O}_{2}^{\prime}\right)$, reduction to $\mathscr{H}_{0} \otimes \mathscr{H}_{0} \subset \mathscr{H} \otimes \mathscr{H}$ and duality, one easily gets $\left(\mathrm{v}^{\prime}\right)$ from $(5.1)$ and $(\mathrm{v}) \Rightarrow\left(\mathrm{v}^{\prime}\right)$.

Conversely if $\left(\mathrm{v}^{\prime}\right)$ holds and $\tau$ is dominant, then $(\mathrm{v})$ holds. It suffices [10] to show that there are normal product states on $\mathfrak{F}\left(\mathcal{O}_{1}\right) \vee \mathfrak{F}^{t}\left(\mathcal{O}_{2}^{\prime}\right)^{-}$, faithful on each algebra.

With $g \in \mathscr{G} \rightarrow \mathscr{V}_{g} \in \mathfrak{F}\left(\mathcal{O}_{2}\right)$ a continuous unitary representation of $\mathscr{G}$ inducing the gauge transformations on $\mathfrak{F}\left(\mathcal{O}_{1}\right), m_{v}$ the normal conditional expectation of $\mathfrak{B}\left(\mathscr{H}_{\pi}\right)$ given by the average over Ad $\mathscr{V}_{g}, g \in \mathscr{G}$, we have for $\psi_{1} \in \mathfrak{F}\left(\mathcal{O}_{1}\right), \psi_{2} \in \mathfrak{F}^{t}\left(\mathcal{O}_{2}^{\prime}\right)^{-}$

$$
m_{v}\left(\psi_{1} \psi_{2}\right)=m\left(\psi_{1}\right) \psi_{2} ; \quad m \circ m_{v}\left(\psi_{1} \psi_{2}\right)=m\left(\psi_{1}\right) m\left(\psi_{2}\right)
$$

with $\omega$ a normal product state over $\mathfrak{U}\left(\mathcal{O}_{1}\right) \vee \mathfrak{U}\left(\mathcal{O}_{2}^{\prime}\right)^{-}, \omega \circ m \circ m_{v}$ is a normal product state over $\mathfrak{F}\left(\mathcal{O}_{1}\right) \vee \mathfrak{F}^{t}\left(\mathcal{O}_{2}^{\prime}\right)^{-}$.

Assumption $\left(\mathrm{v}^{\prime}\right)$ has strong consequences on the structure of $\mathfrak{A}$. With $\mathscr{H}$ a separable Hilbert space and $\mathfrak{B}$ the $C^{*}$-inductive limit of $\mathfrak{B}\left(\mathscr{H}^{\otimes n}\right)$ by $B \in \mathfrak{B}\left(\mathscr{H}^{\otimes n}\right) \rightarrow B \otimes I \in \mathfrak{B}\left(\mathscr{H}^{\otimes n+1}\right)$, for any theory fulfilling $\left(\mathrm{v}^{\prime}\right)$ we have that $\mathfrak{A}$ is isomorphic to $\mathfrak{B}$.

At the local level, duality together with assumption $\left(\mathrm{v}^{\prime}\right)$ imply that $\mathfrak{U}(\mathcal{O})$ is injective. For by duality $\mathfrak{U}(\mathcal{O})=\bigcap_{n} \mathfrak{A}\left(\mathcal{O}_{n}\right)$, if $\mathcal{O}_{n} \in \mathscr{K}, \mathcal{O}_{n+1} \subset \operatorname{Interior}\left(\mathcal{O}_{n}\right)$ and $\bigcap_{n} \mathcal{O}_{n}$ $=0$. With $\mathfrak{M}_{n}$ a type $I$ factor such that

$$
\mathfrak{U}(\mathcal{O})_{n+1} \subset \mathfrak{M}_{n} \subset \mathfrak{U}\left(\mathcal{O}_{n}\right),
$$

we have $\mathfrak{U}(\mathcal{O})=\bigcap_{n} \mathfrak{M}_{n}$ and $\mathfrak{U}(\mathcal{O})$ is injective ${ }^{5}$.

Concerning assumption a) one could argue that, in a Poincaré covariant theory, under an assumption closely related to $\left(\mathrm{v}^{\prime}\right)$ and to the existence of local densities for the generators of Poincaré transformations, any irreducible localized morphism would be covariant.

The other part of a) should be related to additivity (ii) whereby $\mathfrak{A}(\mathcal{O})$ for any small $\mathcal{O}$ generates with its translates all the observables of the theory.

Our last comment is the interpretation of some statements in this paper as Galois relations. With $\mathscr{M} \subset \mathfrak{U}$ and $\mathscr{N} \subset \operatorname{Aut}(\mathfrak{U})$ define $\mathscr{M}^{\perp}=\{\varrho \in \operatorname{Aut}(\mathfrak{U}) / \varrho(A)=A$, $A \in \mathscr{M}\} ; \mathscr{N}^{\perp}=\{A \in \mathfrak{U} / \varrho(A)=A, \varrho \in \mathscr{N}\}$.

Since each $\gamma \in \mathscr{N}$ is locally normal, for any $\mathscr{N} \subset \operatorname{Aut}(\mathfrak{U}), \mathscr{N}^{\perp}$ is a $\mathscr{T}$-closed subalgebra of $\mathfrak{A}$. With $\mathfrak{B} \subset \mathfrak{U}$ a ${ }^{*}$-subalgebra and $\mathscr{A}=\mathfrak{B}^{c}$, it is easily seen that $\mathscr{A} \equiv \mathscr{A}^{\perp \perp}$. Hence by duality

$$
\mathfrak{A}(\mathcal{O})=\mathfrak{U}(\mathcal{O})^{\perp \perp} .
$$

5 R. V. Kadison was the first to note that $\left(\mathrm{v}^{\prime}\right)$ together with continuity would imply hyperfiniteness [21] 
Since $\mathscr{I}(\mathcal{O})^{\perp}=\mathfrak{A}(\mathcal{O})^{c}$, by Proposition 2.3 we also have

$$
\mathscr{I}(\mathcal{O})=\mathscr{I}(\mathcal{O})^{\perp \perp} \text {. }
$$

By definition, $\Gamma(\mathcal{O})=\mathfrak{U}\left(\mathcal{O}^{\prime}\right)^{\perp}$ and $\pi\left(\mathfrak{U}\left(\mathcal{O}^{\prime}\right)^{\perp \perp}\right)=\mathfrak{F}(\mathcal{O})^{\prime} \cap \pi(\mathfrak{U})$. The following relation is a natural conjecture

$$
\mathfrak{U}\left(\mathcal{O}^{\prime}\right)=\mathfrak{U}\left(\mathcal{O}^{\prime}\right)^{\perp \perp} .
$$

By intersection with $\mathfrak{U}\left(\mathcal{O}_{1}\right), \mathcal{O}_{1} \supset \supset \mathcal{O}$, it follows from (5.4)

$$
\left\{\mathfrak{U}(\mathcal{O})^{\prime} \cap \mathfrak{U}\left(\mathcal{O}_{1}\right)\right\}^{\tau}=\mathfrak{U}\left(\mathcal{O}^{\prime}\right) \cap \mathfrak{U}\left(\mathcal{O}_{1}\right) .
$$

Proposition. Let $\mathcal{O}, \mathcal{O}_{1}, \mathcal{O}_{2}$ be double cones, $\mathcal{O} \subset \mathcal{O}_{1} \subset \subset \mathcal{O}_{2}$. Under the assumptions in the Introduction and (5.4), for any choice of unitaries $\mathscr{V}_{g} \in \mathfrak{U}\left(\mathcal{O}_{1}\right)^{\prime} \cap \mathfrak{U}\left(\mathcal{O}_{2}\right)$, such that $\tau_{\xi}\left(\mathscr{V}_{g}\right)=\langle\xi, g\rangle \mathscr{V}_{g}, \xi \in \hat{\mathscr{G}}, g \in \mathscr{G}$, we have

$$
\mathfrak{U}(\mathcal{O})^{c}=\mathfrak{U}\left(\mathcal{O}^{\prime}\right) \stackrel{\mathscr{T}}{\vee}\left\{\mathscr{V}_{g} / g \in \mathscr{G}\right\},
$$

i.e. $\mathfrak{U}(\mathcal{O})^{c}$ is the $\mathscr{T}$-closed ${ }^{*}$-algebra generated by $\mathfrak{U}\left(\mathcal{O}^{\prime}\right)$ and any choice of local charge operators in $\mathfrak{A}(\mathcal{O})^{c}$.

Proof. Since $\mathfrak{A}(\mathcal{O})$ is injective it is generated by an amenable group of unitaries; averaging over the induced automorphism group gives a conditional expectation $\phi$ from $\mathfrak{B}\left(\mathscr{H}_{0}\right)$ onto $\mathfrak{U}(\mathcal{O})^{\prime}$ such that

$$
\phi\left(\mathfrak{U}\left(\mathcal{O}_{1}\right)\right)=\mathfrak{U}\left(\mathcal{O}_{1}\right) \cap \mathfrak{U}(\mathcal{O})^{\prime} \quad \text { for each } \quad \mathcal{O}_{1} \in \mathscr{K}, \quad \mathscr{O} \subset \mathcal{O}_{1} .
$$

Since $\phi(\mathfrak{H})=\mathfrak{A}(\mathcal{O})^{c}$ and $\bigcup_{\mathscr{O}_{1}} \mathfrak{U}\left(\mathcal{O}_{1}\right)$ is norm dense in $\mathfrak{A}$, by norm continuity of $\phi$ also $\bigcup_{\mathcal{O}_{1} \in \mathscr{K}} \mathfrak{A}\left(\mathcal{O}_{1}\right) \cap \mathfrak{A}(\mathcal{O})^{\prime}$ is norm dense in $\mathfrak{A}(\mathcal{O})^{c}$. By Theorem 3.5 and Proposition 2.7, (ii), $\mathfrak{U}(\mathcal{O})^{\prime} \cap \mathfrak{U}\left(\mathcal{O}_{1}\right)$ is generated as a von Neumann algebra by $\mathfrak{U}\left(\mathcal{O}_{1}\right) \cap \mathfrak{U}\left(\mathcal{O}^{\prime}\right)$ and $\mathscr{V}_{g}, g \in \mathscr{G}$. It follows that $\mathfrak{A}(\mathcal{O})^{c} \subset \mathfrak{A}\left(\mathcal{O}^{\prime}\right) \vee^{\mathscr{T}}\left\{\mathscr{V}_{g}, g \in \mathscr{G}\right\}$ and since $\mathfrak{A}(\mathcal{O})^{c}$ is $\mathscr{T}$-closed by the above remarks and $\mathfrak{U}\left(\mathcal{O}^{\prime}\right) \subset \mathfrak{U}(\mathcal{O})^{c}, \mathscr{V}_{g} \in \mathfrak{U}(\mathcal{O})^{c}$, we have also the opposite inclusion and (5.5) is proved.

Assumption a) might be essential for conjecture (5.4). The rest of the paper could easily be modified replacing a) by: there is $\mathcal{O} \in \mathscr{K}$ such that $\Gamma_{C}(\mathcal{O}) \rightarrow \hat{\mathscr{G}}$ is onto. However if such $\mathcal{O}$ cannot be chosen arbitrarily small, there is little hope of recovering charge density Wightman fields.

Note added in proof. In a forthcoming paper in collaboration with Roberto Longo, some results of this paper are generalized and the arguments given here in Sect. 3 are considerably simplified. Dealing with the case where parastatistics are possibly present, i.e. the gauge group $\mathscr{G}$ is not assumed to be abelian, we prove that for each pair of double cones $\mathcal{O}_{1} \subset \subset \mathcal{O}_{2}$ there is a continuous unitary representation $g \rightarrow \mathscr{V}_{g}$ of $\mathscr{G}$ with values in $\mathfrak{F}\left(\mathcal{O}_{2}\right)$ inducing the gauge automorphisms $\alpha_{g}$ on $\mathfrak{F}\left(\mathcal{O}_{1}\right)$ and such that $\alpha_{h}\left(\mathscr{V}_{g}\right)=\mathscr{V}_{h g h^{-1}}, h, g \in G$. If in particular $\mathscr{G}$ is a Lie group the generators of $V$ provide a local current algebra in $\mathfrak{U}\left(\mathcal{O}_{1}\right)^{\prime} \cap \mathfrak{F}\left(\mathcal{O}_{2}\right)$. The center of the von Neumann algebra generated by $\mathscr{V}_{g}, g \in \mathscr{G}$, is an abelian subalgebra of observables in $\mathfrak{U}\left(\mathcal{O}_{1}\right)^{\prime} \cap \mathfrak{U}\left(\mathcal{O}_{2}\right)$, and these observables determine the superselection quantum numbers contained in the region $\mathcal{O}_{1}$ by measurements in the region $\mathcal{O}_{2}$.

Acknowledgement. We would like to thank L. Streit for his kind hospitality at Z.I.F., University of Bielefeld in 1976 when some of the present results were obtained, and D. Kastler for the kind hospitality at the University of Luminy where the paper was eventually written down. We would like to thank Masamichi Takesaki for discussions and explanations on the work [16]. 


\section{References}

1. Haag, R.: Lille Conference 1957 Les problèmes mathématiques de la théorie quantique des champs. CNRS, Paris (1959)

2. Haag, R., Kastler, D.: An algebraic approach to quantum field theory. J. Math. Phys. 5, 848 (1964)

3. Doplicher, S., Haag, R., Roberts, J.E.: Fields, observables and Gauge transformations. I. Commun. Math. Phys. 13, 1 (1969); II. Commun. Math. Phys. 15, 173 (1969)

4. Doplicher, S., Haag, R., Roberts, J.E. : Local observables and particle statistics. I. Commun. Math. Phys. 23, 199 (1971); II. Commun. Math. Phys. 35, 49 (1974)

5. Buchholz, D., Fredenhagen, K.: Locality and the structure of particle states. Commun. Math. Phys. 84, 1-54 (1982)

6. Doplicher, S., Roberts, J.E.: Fields, statistics, and nonabelian Gauge groups. Commun. Math. Phys. 28, 331 (1972)

7. Haag, R.: Bemerkungen zum Nahwirkungsprinzip. Ann. Phys. 7, 29 (1963)

8. Bourbaki, N.: Espaces vectoriels topologiques, fascicule de résultats. Paris: Hermann 1955

9. Haag, R., Schroer, B.: Postulates of quantum field theory. J. Math. Phys. 3, 248 (1962)

10. Buchholz, D.: Product states for local algebras. Commun. Math. Phys. 36, 287 (1974)

11. Summers, S.J. : Normal product states for fermions and twisted duality. (preprint)

12. D'Antoni, C., Longo, R.: Interpolation by type I factors and the flip automorphism. J. Funct. Anal. (to appear)

13. Takesaki, M. : Duality for crossed products and the structure of von Neumann algebras of type III. Acta Math. 131, 249 (1974)

14. Borchers, H.J.: A remark on a theorem of Misra. Commun. Math. Phys. 4, 315 (1967)

15. Dixmier, J.: Les algébres d'operateurs dans l'Espace Hilbertien. Paris: Gauthier-Villars 1969

16. Connes, A., Takesaki, M.: The flow of weights on factors of type III. Tohoku Math. J. 29, 473 (1977)

17. Wightman, A.S.: Ann. Inst. Henry Poincaré 1, 403 (1964)

18. Driessler, W.: Duality and absence of locally generated superselection sectors. Commun. Math. Phys. 70, 213 (1979)

19. Roberts, J.E.: Local cohomology and superselection sectors. Commun. Math. Phys. 51, 107 (1976)

20. Haag, R., Kadison, R.V., Kastler, D. : Nets of $C^{*}$-algebras and classification of states. Commun. Math. Phys. 16, 11 (1970)

21. Kadison, R.V.: Private communication

22. Longo, R.: Algebraic and modular structure of von Neumann algebras of physics. Proceedings A.M.S. Summer Institute on Operator Algebras, Kingston, 1981

Communicated by R. Jost

Received November 17, 1981 